\title{
Case report on the treatment of atrial fibrillation in a pregnant woman of 33 weeks' gestation
}

\author{
N. Murphy ${ }^{1}$, D. Sugrue ${ }^{2}$, P. McKenna ${ }^{1}$ \\ ${ }^{1}$ Rotunda Hospital, Dublin, Ireland \\ ${ }^{2}$ Mater Misericordiae Hospital, Dublin, Ireland \\ Email: niamhcmurphy@gmail.com
}

Received 30 November 2013; revised 20 December 2013; accepted 28 December 2013

Copyright (C) 2014 N. Murphy et al. This is an open access article distributed under the Creative Commons Attribution License, which permits unrestricted use, distribution, and reproduction in any medium, provided the original work is properly cited. In accordance of the Creative Commons Attribution License all Copyrights (C) 2014 are reserved for SCIRP and the owner of the intellectual property N. Murphy et al. All Copyright (C) 2014 are guarded by law and by SCIRP as a guardian.

\section{ABSTRACT}

This case report highlights a recent case in the Rotunda Hospital of a patient of 33 weeks' gestation with fast atrial fibrillation. She had an unusual presentation given that she had no prior cardiac history or obvious aetiology. She underwent successful DC cardioversion and was well on discharge. Follow-up with cardiology has been arranged.

\section{KEYWORDS}

\section{Atrial Fibrillation; Pregnancy; Cardioversion}

\section{INTRODUCTION}

This case involved the treatment of atrial fibrillation in a woman of 33 weeks' gestation. There was no prior cardiac history. The only potential cause identified was infection given that she was being treated for a urinary tract infection.

Atrial fibrillation is the most common cardiac arrhythmia. It may cause no symptoms but is often associated with palpitations, chest pain or congestive cardiac failure. Patients are at risk of a thromboemoblic event. It can be managed by either controlling rate or rhythm with no difference in stroke risk between the treatments [1].

Rate control is often commenced at the time of diagnosis. This is done to decrease the heart rate but does not normalize the heart rhythm [2].

Physiologic changes of pregnancy can predispose women to cardiac arrhythmias however atrial fibrillation is still rare and usually occurs in women with underlying cardiac arrhythmias. Diagnosis requires exclusion of all possible aetiologies. Electical cardioversion as opposed

*Corresponding author. to chemical cardioversion is the treatment of choice and pregnant women also require fetal growth surveillance and antenatal testing in persistent atrial fibrillation [3].

\section{CASE PRESENTATION}

AMcM was a 37-year-old female in her third pregnancy.

She presented to the Emergency Room of a general hospital complaining of flu like symptoms. At the time of presentation, she was 32 weeks pregnant with dichorionicdiamniotic twins.

She was diagnosed with a urinary tract infection, commenced on po cefuroxime, rehydrated in the ER and discharged.

She represented three days later complaining of sudden onset palpitations. There was no associated chest pain or dyspnoea. Electrocardiogram confirmed new onset fast atrial fibrillation.

She was admitted to the Coronary Care Unit. Medications were commenced including Metoprolol $50 \mathrm{mg}$ od and Enoxaparin $1 \mathrm{mg} / \mathrm{kg}$.

Echocardiogram was performed the following day. This showed mild mitral regurgitation with no other significant findings. In particular, there was no evidence of an atrial septal defect and there was no thrombus visualized in the left atrium.

The next day, she was transferred to our tertiary referral unit for further assessment and management. She was admitted to the High Dependency Unit for monitoring. Since commencing beta blockers, she experienced no further palpitations. ECG still confirmed the presence of Atrial Fibrillation (Figure 1). CTG monitoring and ultrasound scan confirmed normal biophysical profiles and Doppler flow in both twins.

She underwent cardioversion by the cardiology team. A consultant obstetrician was also present to intervene if 
there were unforeseen obstetric complications. Synchonised electrical cardioversion was used and the current delivered during the $\mathrm{R}$ wave of the QRS complex. Sinus rhythm was obtained

MrsMcM remained well in the unit over the following four days. Twice daily ECGs confirmed sinus rhythm on each occasion (Figure 2). There were no further episodes of palpitations. MrsMcM was discharged and attended for outpatient cardiology care in her local hospital. She had an induction of labour with vaginal delivery at 38 weeks gestation

\section{DISCUSSION}

MrsMcM was correctly diagnosed with atrial fibrillation and treated appropriately. The risk of systemic embolisation in atrial fibrillation during pregnancy is probably due to circulatory statis in the atrium and left atrial appendage, and the hyperviscosity and hypercoagulable state of pregnancy with subsequent thrombus formation. The management of atrial fibrillation in haemodynamically stable pregnant women initially involves control of the

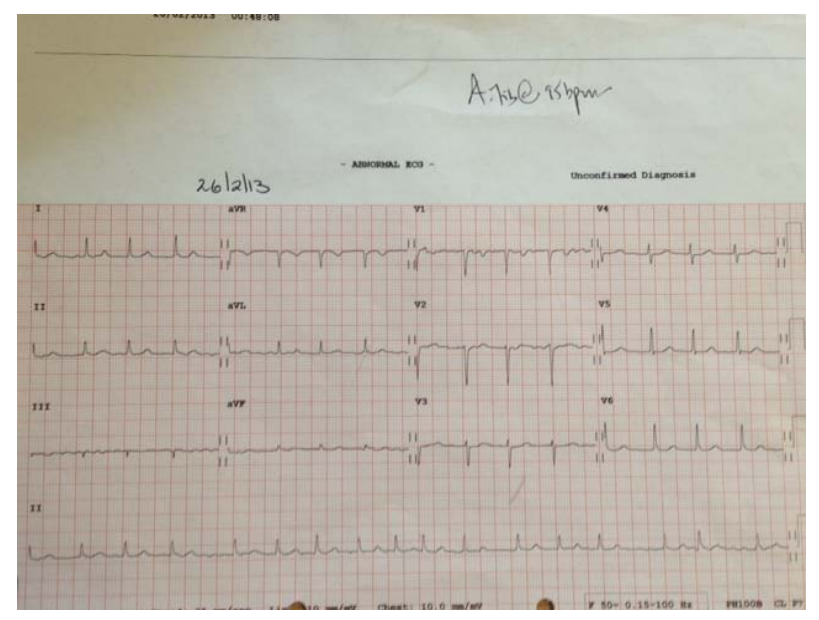

Figure 1. ECG on admission to the Rotunda Hospital.

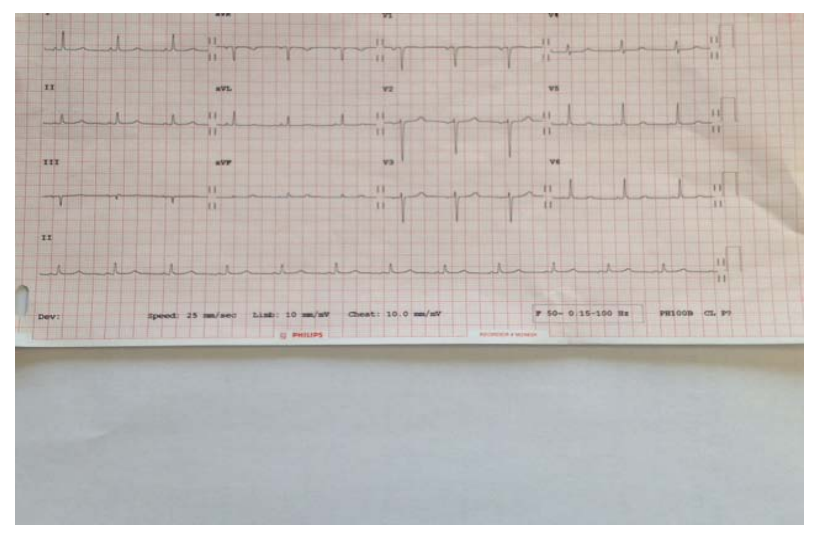

Figure 2. ECG in Rotunda Hospital post electrical cardioversion. ventricular rate by digoxin, a beta blocker or a calcium channel blocker or a combination of these. In elective situations, rate should be controlled initially and this can later be followed by rhythm control using DC cardioversion [4].

It is rare for new onset atrial fibrillation to occur in pregnancy with no aetiology for same. Cases in the literature highlighted that most of these cases involve women with known pre-existing cardiac disease or where investigations confirm cardiac disease. In our case, echocardiogram was essentially normal. The only potential cause found is infection given that she was being treated for a urinary tract infection. We have found no cases such as ours in the Irish literature.

\section{REFERENCES}

[1] Wyse et al. (2002) A comparison of rate control and rhythm control in patients with atrial fibrillation. New England Journal of Medicine, 347, 1825-1833.

[2] National Collaborating Centre for Chronic Conditions (2006) Atrial fibrillation: National clinical guideline for management in primary and secondary care. Royal College of Physicians, London.

[3] DiCarlo-Meacham et al. (2011) Atrial fibrillation in pregnancy, obstetrics and gynaecology. 117, 489-492.

[4] Niwa, K. and Tateno, S. (2006) Maternal cardiac arrhythmias. In: Steer, P.J., Gatzoulis, M.A. and Baker, P., Eds., Heart Disease and Pregnancy, RCOG Press, London, 254-255. 